Paediatr Paedolog 2017 · 52:216-219

DOI 10.1007/s00608-017-0490-0

Online publiziert: 25. Juli 2017

(c) Der/die Autor(en) 2017. Dieser Artikel ist

eine Open-Access-Publikation.

CrossMark

P. Scheer

Graz, Österreich

\title{
Fehlermanagement in der pädiatrischen Praxis aus der Sicht des Gutachters
}

schwächen, wenn sie passiert sind? Zuletzt: Wie kann man sich selbst vor den Folgen schützen, wenn man sich denn traut auch zu diesem berechtigten Anliegen zu stehen?

In meiner Vorbereitung dieser Überlegungen bin ich zuerst klassische Wege gegangen: ich habe Gesetze gelesen und Definitionen des Kunstfehlers, habe mich an meine eigenen Kasuistiken erinnert, an die Fälle, die ich bei Gericht bearbeitet habe und zwar in den verschiedensten gesetzlichen Umfeldern.

Dann ist mir aber klargeworden, dass das zu spät ansetzt. Wenn der Fehler schon geschehen ist, wenn nicht nur ein kindlicher Patient geschädigt wurde, sondern die Sache so schlimm war oder so schlecht gemanagt wurde, dass sie gerichtsanhängig wurde, dann ist ja schon viel schiefgegangen. Sowohl der einfache Satz (Murphy's law): „Alles, was schiefgehen kann, wird auch schiefgehen" [1], den er dem Bereich des Flugzeugbaus zugeschrieben hat, gibt da schon viel mehr Informationen her als das Gesetz oder die Legislatur.

Ich habe mich daher entschlossen, meine Ausführungen auf die Punkte

1. Häufige Fehlerursachen (•Tab. 1) sowie

2. Fehlerbewältigung zu beschränken. sagen die Einen: „Beim Arzt ist das was Besonderes. Dem darf so was nicht passieren, da geht's doch um Menschenleben!" Die Anderen aber sind verständiger und wissen, dass Ärzten Fehler passieren können. Was kann und soll man aber machen, um Fehler zu vermeiden? Was soll man tun, um deren Folgen abzu-

Nach einer Fortbildung der steirischen Kinderund Jugendärzte am 19.10.2016 in Graz

\section{Stress}

Hans Selye (1907-82), dem aus dem ungarischen Teil des alten Österreich stammenden, außergewöhnlichen Forscher, fiel als junger Arzt in einer Ambulanz auf, dass man echte Kranke von Gesunden am Aussehen unterscheiden könne. So entdeckte er nach und nach die
Stressreaktion, die selbst der Einzeller, dessen Umgebungsflüssigkeit man Salz zusetzt, zeigt. Stress ist die Reaktion jedes Organismus auf Herausforderungen und unbedingt erforderlich, um diesen gewachsen zu sein.

Im Alltag wird unter Stress allerdings eine übermäßige Herausforderung verstanden, die an einen gestellt wird. Jeder empfindet Stress anders und bei jedem lösen andere Faktoren Stress aus. Interessant in diesem Zusammenhang ist eine Studie, die an erfahrenen und unerfahrenen Geburtshelfern gemacht wurde. Im Nachtdienst wurden die Stressindikatoren nach der Berufung zu einer Sectio Caesarea beobachtet und festgestellt, dass die Angehörigen beider Gruppen gleich viel Stress hatten, nur die erfahrenen Geburtshelfer ihn nicht mehr spürten und angaben, dass eine Sectio sie nicht stresst. Das ist kein gutes Zeichen, denn das zeigt, dass die erfahreneren Ärzte nicht stressresistenter wurden, sondern nur ihre Wahrnehmung schlechter. Das passt zu anderen Studien, die zeigen, dass selbst ein Nachtdienst ohne besondere Vorkommnisse und mit der Möglichkeit zu schlafen keine Reduktion der Stressindikatoren (Hautwiderstand, Pulsfrequenz, Atemfrequenz etc.) bringt. Stressresistenz ist nicht erlernbar.

Deshalb werden Angehörige von Eli- tetruppen danach ausgesucht, ob sie

\section{Tab. 1 Fehlerursachen}
1. Stress
2. Arbeitsüberlastung
3. Inkompetenz
4. Konzentrationsmangel, oder -verlust
5. Kommunikationsschwierigkeiten 
Stress besser oder schlechter aushalten. Generell kann man sagen, dass empfindsame Menschen meist wenig Stress aushalten und dass sog. starke Männer, die Stress weder aussprechen, noch wahrnehmen können, nicht stressresistent sind.

\section{Stressresistenz}

Die eigene Stressresistenz kann man gut testen, indem man einen normalen Tag ansieht und sich fragt, was einen mitnimmt, erschöpft, aufregt, und dann die Zeitspanne, die bis zur Beruhigung benötigt wird, misst. Sie soll keinesfalls über 20 min andauern. Die Wiederherstellung des Status quo ante wird als Resilienz bezeichnet - ein Begriff, der aus der Reifentechnologie kommt. Er meint jene Zeit, die ein luftgefüllter Reifen benötigt, um nach einer Verformung wieder seine alte, runde Form anzunehmen. In der Psychologie wird dieser technische Vergleich auf die Wiedererreichung der Funktionsfähigkeit nach einer Verletzung oder einer Herausforderung angewandt.

Unter Stress, so behaupten die Beobachter von Piloten während ihres Einsatzes, wird alle drei Minuten ein Fehler gemacht. Wobei unter Fehler eine Handlung verstanden wird, die entweder unnötig oder sogar dem Flugbetrieb nicht dienlich ist. Das kann durch Monitoring des Verhaltens von Piloten festgehalten werden. Im Gegensatz dazu werden in nicht stressigen Situation nur stündlich Fehler gemacht.

\section{Stressoren}

Wenn ein Arzt durch das volle Wartezimmer gestresst wird, dann muss versucht werden, das $\mathrm{zu}$ verhindern versuchen. Sich einzureden, dass man sich daran gewöhnt hätte, nützt nichts.

Stress kann auch durch Situationen ausgelöst werden, die für andere nicht nachvollziehbar sind. Da genügt eine falsche Zureichung, eine wenig vertraute Ordinationshilfe, Anrufe, die man so oder zu diesem Zeitpunkt nicht entgegennehmen will, ungeliebte Gerüche, Erinnerungen, die durch einen Geruch, oder eine Geste ausgelöst werden, und vieles andere mehr. All das ist nicht $\mathrm{zu}$ verhindern. Einfach gesagt: Ein Mensch ist umso resilienter, je mehr er sich die Irritationen eingesteht. Das ist aber kulturspezifisch ungewöhnlich. An sich gestehen wir uns gar nichts ein, und wenn, dann höchstens heimlich.

\section{Kulturspezifische Schmerz- unterdrückung}

Es beginnt bei dummen Aussagen wie „Ein Indianer kennt keinen Schmerz!“, zieht sich über dumme Belobigungen bei Kindern weiter, wenn sie eine Impfung oder eine Blutabnahme ohne Weinen überstehen, und endet schließlich bei den Fähnchen, die man auch in Abteilungen für Kinderchirurgie sieht, wo dem Kind zu seiner Tapferkeit gratuliert wird. Dies sind alles Maßnahmen zum frühzeitigen Lehren kulturspezifischer Schmerzunterdrückung und machen Kinder unfähig, über ihren Schmerz zu sprechen. Aldous Huxley hat in seinem Spätwerk Eiland der anderen der die Resilienz fördernden Verarbeitung das Wort gesprochen: Der Held, der nach einem Schiffbruch an Land geschwemmt wird und sich mit letzter Kraft die Klippe hochzieht, wird von einem Papagei empfangen, der ihn auffordert, immer wieder und immer wieder seine Geschichte zu erzählen, bis der Mann selbst zu schmunzeln beginnt.

\section{Kommunikation}

Kommen wir zu den Kommunikationsschwierigkeiten. Diese können und werden überall auftreten. Wenn man ein Chef, eine Chefin ist, hat man Kommunikation über Fehler vorzuleben.

Damit sind wir beim zweiten Punkt, der unter dem Namen „crew ressource management" (CRM) in der Luftfahrt Bedeutung erlangt hat. Sie besagt, dass die Kommunikation von Fehlern gefördert werden muss und dass diese wichtiger ist als Hierarchie und Ordentlichkeit. Unterdrückte Fehlermeldungen sind zu gefährlich, als dass sie zugelassen werden können. Bestraft wird nicht die Meldung, sondern allenfalls deren Verzögerung oder ängstliche Unterdrückung [2].

Kommunikation in einer Praxis und eine vertrauensvolle Zusammenarbeit sind fast am wichtigsten, sowohl im prophylaktischen als auch im kurativen Fehlermanagement. Jedem und jeder müssen Fehler gestattet sein. Jeder Mensch macht Fehler, daher darf jeder mögliche Fehler immer passieren. Beobachtungen von Fehlern müssen mitgeteilt werden und sind grundsätzlich nicht Teil einer hierarchischen Kommunikation.

Eine z. B. neueingetretene Hilfs- oder Reinigungskraft darf und muss Fehler sehen und ansprechen können. Das beginnt bei der Hygiene, darf aber auch Fragen des Patientenmanagements erfassen (Wie lange wartet wer? Wie wird das gesteuert?) und letztlich darf und soll eine solche Kraft auch Fehler des eingesessenen Personals ansprechen dürfen.

In meiner Ausbildungszeit war mein Ausbildungsassistent Sohn eines einflussreichen österreichischen Politikers. Leider war er alkoholkrank. Das Ansprechen dieser Störung führte nicht zu einer Änderung des Einsatzes dieses Arztes. Erst ein Missgeschick im Dienst an einer anderen Abteilung führte zur Freisetzung des Kollegen.

Kommunikation und deren Förderung ist das Um und Auf des Fehlermanagements. Stress- und angstfrei muss sie sein. Mitarbeiter sind alle gleich viel Wert; Bedrohungen gehen von ängstlichen und nicht von mutigen Mitarbeitern aus. Kritik muss erlaubt sein und gefördert werden.

\section{Inkompetenz}

Somit komme ich zum gefährlichsten Punkt der prophylaktischen Fehlervermeidung: die Selbstüberschätzung. Für die Diplomarbeit Benjamin Rettls [3] hat Univ.-Lektor Dr. Dr. Peter Voitl angeregt zu untersuchen, welche Notfallausrüstung niedergelassene Kinderärzte in der Praxis haben und welche Eingriffe sie sich im Notfall zutrauen. Allein die Tatsache, dass sich fast $90 \%$ das Anlegen eines intraossären Zugangs zutrauten, aber nur $20 \%$ die Ausrüstung hatten, zeigte schon Selbstüberschätzung. Die Zusatzfrage, wie oft und wann zuletzt sie das gemacht hatten (inklusive Simulation an einer Puppe) eröffnete eine dramatische Innensicht: Keine des teilnehmenden, re- 
präsentativen Forums an Kinderärzten im Raum Wien und Umgebung hatte einen derartigen Eingriff in den letzten 20 Jahren durchgeführt.

Die Diplomarbeit ist ein Fundus an derartigen Widersprüchen. Ärzte trauen sich Eingriffe und Wiederbelebungsmaßnahmen zu, die sie entweder nie gemacht haben oder schon sehr, sehr lange nicht. Selbst eine kardiopulmonale Reanimation, die eigentlich jeder können sollte, der einen Führerscheinkurs absolviert hat, muss immer wieder geübt werden.

Bei der feuerpolizeilichen Abnahme von Gebäuden sind Vorschriften gegeben und werden eingehalten. Die Wahrheit zeigt sich aber immer erst, wenn während einer Übung ein Brandfall simuliert wird. Dann zeigt sich, dass etwa schöne Glasscheiben bei Verrauchung von den eingreifenden Feuerwehrmännern nicht gesehen werden können und sie trotz Kopflicht dagegenlaufen. So konnten die gespielten Patienten der geschützten Abteilung der neugebauten, psychiatrischen Abteilung des LKH Graz nicht gerettet werden. Das Anbringen von zwar nicht so schönen, aber reflektierenden Streifen in Augenhöhe veränderte diesen Fehler nachhaltig.

\section{Kleine Fehler, große Fehler}

Der Fehler muss gesagt, besprochen und analysiert werden. Das gilt sowohl für akute, kleine Fehler als auch für systemimmanente Fehler und sog. schlechte Gewohnheiten.

In einem Gutachten wurde ich gefragt, $o b$ der Tod eines fast 3-jährigen Kindes zu verhindern gewesen wäre. Das Kind war nach mehreren ambulanten Betreuungen aufgenommen worden. Der behandelnde Oberarzt hatte eine Leukopenie im Blutbild gesehen und den richtigen Schluss gezogen, dass die lange Anamnese zu einem Aufbrauchen der Leukozyten geführt haben musste. Allerdings sah er sich außerstande, das Blutbild selbst anzusehen. Stattdessen versuchte er eine Vorstellung an einer hämatologischen Spezialabteilung zu veranlassen, die ihm in 3 Tagen in Aussicht gestellt wurde. Nach meiner Ansicht hätte er bei der Wahrnehmung sei-

Paediatr Paedolog 2017 · 52:216-219 DOI 10.1007/s00608-017-0490-0

(c) Der/die Autor(en) 2017. Dieser Artikel ist eine Open-Access-Publikation.

\section{P. Scheer \\ Fehlermanagement in der pädiatrischen Praxis aus der Sicht des Gutachters}

\section{Zusammenfassung}

Fehlermanagement stellt eine prophylaktische Maßnahme dar, die in jeder Ordination eines Kinder- und Jugendarzt seinen Platz haben sollte. Bekannte und eher einfache Maßnahmen zur Reduktion der Wahrscheinlichkeit des Auftretens von Fehlern sind Stressmanagement und die Vermeidung von Arbeitsüberlastung. Schon komplexer wird es, wenn man sich mit der Kommunikation in der Praxis und der eigenen Selbstüberschätzung zu befassen beginnt. Aus der Analyse von Fehlern, die zum Teil auch gerichtsanhängig wurden, wird ersichtlich, dass die mangelnde Kommunikation in der Praxis im Dreieck zwischen Kind, Eltern und Arzt bzw. Arzthelfer ein Hauptauslöser von Fehlern ist. Eine Diplomarbeit, durchgeführt an der medizinischen Universität Wien, zeigt darüber hinaus, dass mangelnde Selbsteinschätzung ebenso fatal sein kann. Benjamin Rettl hat in etwa 40 Praxen einerseits die Kenntnisse von Notfalleingriffen abgefragt und anderseits die vorhandene Ausrüstung kontrolliert. Es zeigte sich, dass Ärzte sich sehr viel mehr zutrauen, als sie vorhalten, und noch mehr als sie regelmäßig üben. Bescheidenheit und Demut sind gefragt, wenn die Kommunikation in der Praxis so verbessert werden soll, wie es die Fluggesellschaften seit vielen Jahren machen: Jeder ist gleichberechtigt, wenn es um die Wahrnehmung und Verhinderung von Fehlern geht. Zuletzt noch eines: Zu einem Fehler stehen, ihn eingestehen und sich entschuldigen, gehört zu den vornehmsten Aufgaben eines Arztes.

\section{Schlüsselwörter}

Fehlermanagement · Pädiatrische Praxis . Notfallausrüstung · Kommunikation . Fähigkeiten

\section{Malpractice management in pediatric practice from the viewpoint of the consultant}

\section{Abstract}

The management of medical malpractice is highlighted, in pediatrics in particular, this should have a place in every intervention performed by a pediatric physician. Simple, well-known measures to reduce the probability of the occurrence of causes of malpractice are stress management and avoidance of work overload. The matter becomes even more complex if we start to consider communication in the practice and pediatricians own overconfidence. From the analysis of malpractice, which was partly addressed in court, it is obvious that the lack of communication in the triangle of child, parents, and physicians or medical personnel is the main trigger for malpractice. Furthermore, a doctoral thesis carried out at the Medical University of Vienna, shows that lack of knowledge about one's skills can be just as fatal. Benjamin Rettl queried around 40 practices with regard to the emergency resuscitation they are able to administer and examined the equipment available. He showed that the confidence that physicians have in themselves is much greater than might be their ability, and even more than their regularly practice. Modesty and humility are required if communication in the practice is to be improved, which airlines have done for many years: everyone is equal when it comes to the awareness and prevention of malpractice. Lastly if malpractice occurs one has to admit it and to apologise for it.

\section{Keywords}

Prevention of malpractice - Pediatric practice . Emergency interventions - Communication . Skills ner begrenzten Kompetenz („Kann kein Blutbild im Mikroskop beurteilen") den Patienten wegen seines Mangels überweisen müssen. Das wäre zwar menschlich schmerzlich gewesen, hätte aber wahrscheinlich das Leben des Kindes gerettet.
Die Selbstüberschätzung, kombiniert mit einer Furcht vor Kritik gehört zu jenen Amalgamen, die zu einem echten Fehler führen können. 


\section{Fehlerbewältigung}

Zum Abschluss noch ein Wort zum Einstehen: Das ist ein weitgehend vergessener Begriff. Er bedeutet, dass man zu seinen Taten steht. Er war, so fürchte ich, zuletzt im Zusammenhang mit Ehre und Treue oder anderen sog. Tugenden soldatischen Lebens vertraut. Ich weine denen nicht nach, wenngleich ich bezweifle, dass die Menschheit einem friedlichen Jahrhundert entgegengeht. Meine Generation hat 70 Jahre Frieden in Europa erleben dürfen, wohingegen an vielen Orten ungezählte Kriege stattfanden. Die Tugend des Einstehens allerdings ist zu Unrecht vergessen worden. Sie bedeutet, dass man zu seinem Fehler steht, sich entschuldigt und bereit ist, die Konsequenzen zu tragen.

Sollte aber ein Fehler eingetreten und nicht wiedergutzumachen sein, wie es etwa der Tod durch einen Fehler ist, dann geht es nicht nur darum, die eigene Haut zu retten, sondern vor allem einzustehen, zuzugeben und den Ärger und die Wut der Betroffenen auszuhalten. Ich weiß wohl, dass Versicherungen davon abraten. Sie fürchten das Prozessrisiko und machen dem Arzt Angst. Angst vor Deckungsverlust und ähnlichem. Vor allem sollte es jedoch meiner Meinung nach um die Reduktion des Schmerzes der Angehörigen und um die Wiederherstellung des eigenen Lebensgefühls gehen. Das mag idealistisch sein.

\section{Schluss}

Damit ist alles gesagt. Fehlermanagement enthält Selbsteinschätzung, Kommunikation und Einstehen. Fehlt einer dieser Faktoren, so können schwerwiegende Fehler geschehen, die Menschen das Leben kosten können.

\section{Fazit für die Praxis}

Fehlermanagement in der pädiatrischen Praxis besteht aus: Stressreduktion, Selbsteinschätzung und Kommunikation. Alle drei bedürfen der Pflege und Aufmerksamkeit. Es wird empfohlen, sich allen drei Bereichen zu Zeiten, in denen Ruhe und Geduld herrscht, zuzuwenden. Vor allem die Selbsterflexion im Team und die Bescheidenheit dessen, was diese Praxis leisten kann, scheint bedeutsam.

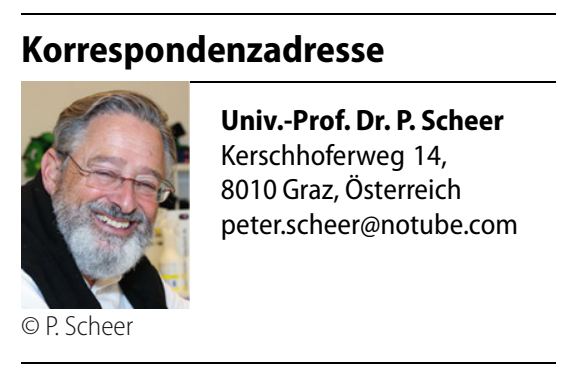

Danksagung. Open access funding provided by Medical University of Graz. Besonderer Dank gilt Herrn Prim. Univ.-Lekt. Dr. Dr. P. Voitl, Wien, für die Hilfe bei der Vorbereitung sowie der stellvertretenden Fachgruppenobfrau Steiermark Frau Dr. G. Kaltenbäck, Graz, für die Einladung und Unterstützung bei der Vorbereitung.

\section{Einhaltung ethischer Richtlinien}

Interessenkonflikt. P. Scheer gibt an, dass kein Interessenkonflikt besteht.
Dieser Beitrag beinhaltet keine von den Autoren durchgeführten Studien an Menschen oder Tieren.

Open Access. Dieser Artikel wird unter der Creative Commons Namensnennung 4.0 International Lizenz (http://creativecommons.org/licenses/by/4.0/deed. de) veröffentlicht, welche die Nutzung, Vervielfältigung, Bearbeitung, Verbreitung und Wiedergabe in jeglichem Medium und Format erlaubt, sofern Sie den/die ursprünglichen Autor(en) und die Quelle ordnungsgemäßnennen, einen Linkzur Creative Commons Lizenz beifügen und angeben, ob Änderungen vorgenommen wurden.

\section{Literatur}

1. Nach Wikipedia. https://de.wikipedia.org/wiki/ Murphys_Gesetz.Zugegriffen: 15.10.2016

2. Graven J (2015) Interview mit dem ehemaligen Piloten und Managertrainer P.-K. Brandl. https://www.impulse.de/management/ das-verschweigen-von-fehlern-muss-zurtodsuende-werden/2025441.html. Zugegriffen: 16. Okt. 16

3. Rettl B (2016) Notfallmanagement in der pädiatrischen Praxis. Diplomarbeit zur Erlangung des akademischen Grades: Dr. med. univ. an der Medizinischen Uni Wien, Jänner 2016.
Hier steht eine Anzeige.

\section{首 Springer}

